\title{
Inducible expression of a fusion gene encoding two proteinase inhibitors leads to insect and pathogen resistance in transgenic rice
}

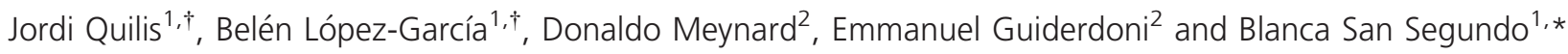 \\ ${ }^{1}$ Centre for Research in Agricultural Genomics (CRAG), CSIC-IRTA-UAB-UB, Edifici CRAG, Barcelona, Spain \\ ${ }^{2}$ CIRAD, UMR AGAP, Montpellier Cedex 5, France
}

Received 11 June 2013,

revised 10 September 2013

accepted 14 October 2013.

*Correspondence (Tel +34 935636600;

fax +34 935636601;

email blanca.sansegundo@cragenomica.es)

${ }^{\dagger}$ These authors have contributed equally to this work.

Keywords: Chilo suppressalis, Fusion protein, Magnaporthe oryzae, Maize proteinase inhibitor, Oryza sativa, Potato carboxypeptidase inhibitor.

\begin{abstract}
Summary
Plant proteinase inhibitors (PIs) are considered as candidates for increased insect resistance in transgenic plants. Insect adaptation to PI ingestion might, however, compromise the benefits received by transgenic expression of Pls. In this study, the maize proteinase inhibitor (MPI), an inhibitor of insect serine proteinases, and the potato carboxypeptidase inhibitor (PCI) were fused into a single open reading frame and introduced into rice plants. The two Pls were linked using either the processing site of the Bacillus thuringiensis Cry1B precursor protein or the 2A sequence from the foot-and-mouth disease virus (FMDV). Expression of each fusion gene was driven by the wound- and pathogen-inducible mpi promoter. The mpi-pci fusion gene was stably inherited for at least three generations with no penalty on plant phenotype. An important reduction in larval weight of Chilo suppressalis fed on mpi-pci rice, compared with larvae fed on wild-type plants, was observed. Expression of the mpi-pci fusion gene confers resistance to C. suppressalis (striped stem borer), one of the most important insect pest of rice. The mpi-pci expression systems described may represent a suitable strategy for insect pest control, better than strategies based on the use of single PI genes, by preventing insect adaptive responses. The rice plants expressing the mpi-pci fusion gene also showed enhanced resistance to infection by the fungus Magnaporthe oryzae, the causal agent of the rice blast disease. Our results illustrate the usefulness of the inducible expression of the mpi-pci fusion gene for dual resistance against insects and pathogens in rice plants.
\end{abstract}

\section{Introduction}

Rice (Oryza sativa L.) is one of the most important cereal crops in the world and a source of food for more than half of the world's population. Rice yields can be severely compromised by the lepidopteran insect Chilo suppressalis (striped stem borer) (Dale, 1994). Strategies for controlling this pest based on the use of chemical insecticides are not effective for the control of C. suppressalis because larvae enter shortly after hatching into the plant and are protected from the effect of insecticide treatment. Moreover, the repeated use of agrochemicals for the control of C. suppressalis has several drawbacks, including the lack of specificity, the possible effects on beneficial organisms, the incidence of resistance development upon prolonged application and the adverse impact on human health and the environment.

An alternative approach for the control of $C$. suppressalis is the development of genetically modified rice plants expressing insecticidal genes. Along with this, many crop plants expressing $B t$ genes from the soil bacterium Bacillus thuringiensis (encoding $\delta$-endotoxins, also known as Cry proteins) that show insect resistance have been developed (Sanahuja et al., 2011; Stevens et al., 2012). Knowing the ability of insects to develop resistance against insecticidal compounds and that insect control products based on topically applied Bt toxins are widely used, it is increasingly clear that alternative or complementary control strategies must by developed to assure that we do not compromise the benefits provided by Bt-based insect pest control strategies.
Plant proteinase inhibitors (PIs) have been also identified as candidates for the development of insect-resistant transgenic crops. Plant Pls are usually present in reproductive tissues (seeds, tubers) where they are synthesized and stored (Koiwa et al., 1997; Ryan, 1990). Furthermore, Pl gene expression is induced in vegetative tissues by chewing insects and wounding. The usefulness of plant Pls to reduce insect attack in controlled greenhouse conditions and in the field is also documented (Duan et al., 1996; Dunse et al., 2010; Haq et al., 2004; Hilder et al., 1987; Johnson et al., 1989; Stevens et al., 2012; Vila et al., 2005). Moreover, the use of Pls has been regarded as a suitable alternative to Bt in pest control, as milder toxicity of these genes is expected to exert a lower selection pressure to the insect.

Plant Pls function in the plant defence response against herbivorous insects via the inhibition of insect digestive proteinases (Broadway, 1996; Johnson et al., 1989; Srinivasan et al., 2005). When plant Pls bind to the target proteinases, they block the digestion of proteins, leading to growth and developmental delays, and increased mortality. However, insects are able to adapt to the presence of inhibitors in the diet by either overproducing Pl-sensitive proteinases, replacing the inhibited enzymes by Pl-insensitive proteinases, or inducing the production of PI-degrading enzymes (Broadway, 1997; Gatehouse, 2002; Girard et al., 1998a; Giri et al., 1998; Jongsma et al., 1995). A phenomenon of overcompensation to dietary proteinase inhibitors produced in transgenic plants resulting in increased weight gain of the target pest has also been described (Cloutier et al., 2000; De Leo et al., 1998; Girard et al., 1998b). Most of these 
problems can be associated with the use of a single PI transgene targeting only one class of proteases in the insect midgut. In this context, the simultaneous expression of Pls with different modes of action in transgenic plants not only might confer increased level of protection against insect attack but also would hinder insect adaptation to PI ingestion.

We previously reported that wounding and insect feeding induce the expression of the mpi (maize proteinase inhibitor) gene in maize plants (Cordero et al., 1994). MPI is a bifunctional inhibitor that effectively inhibits digestive serine proteinases, namely elastase and chymotrypsin proteinases from the lepidopteran insects Spodoptera littoralis and Chilo suppressalis (Tamayo et al., 2000; Vila et al., 2005). In addition to wounding, mpi expression is induced by fungal infection in maize plants (Cordero et al., 1994). In this respect, several proteinase inhibitors have been reported to inhibit the in vitro growth of phytopathogenic fungi, and, for some of them, a defence role in resistance to fungal pathogens has been proposed (Haq et al., 2004; Kim et al., 2009; Laluk and Mengiste, 2011). However, despite the body of information currently available on plant proteinase inhibitors as insecticidal agents, their potential role as antimicrobial agents remains less explored.

On the other hand, the potato carboxypeptidase inhibitor (PCI) accumulates in potato tubers as well as in wounded leaves of potato plants (Graham and Ryan, 1981; Villanueva et al., 1998). $\mathrm{PCl}$ inhibits digestive carboxypeptidase activities from the lepidopteran insect Helicoverpa armigera (Bayés et al., 2003). However, the constitutive expression of pci in rice has an apparent stimulatory effect on the growth of $C$. suppressalis larvae fed on the pci transgenic plants, suggesting an adaptive mechanism in the larval gut to compensate for the effect of $\mathrm{PCl}$ ingestion (Quilis et al., 2007).

In this study, we examined the effect of expression of a fusion gene consisting of two plant proteinase inhibitors, mpi and pci, in transgenic rice focusing on resistance against insect attack and pathogen infection. Two different strategies were assayed for obtaining the fusion gene depending on the sequence used to fuse the two individual $P$ genes in the form of a single transcriptional unit. In both cases, the expression of the mpi-pci fusion gene is driven by the wound- and pathogen-inducible $m p i$ promoter. We report that the inducible expression of the mpi-pci fusion gene confers resistance to the lepidopteran insect $C$. suppressalis as well as to the fungal pathogen Magnaporthe oryzae, the causal agent of the rice blast disease.

\section{Results}

\section{Production of transgenic rice plants expressing a mpi-pci fusion gene}

Two different constructs containing a mpi-pci hybrid gene that differed on the linker sequence used to fuse the two genes were prepared (Figure 1a; Methods S1). The first strategy was selected based on the potential of the FMDV2A (foot-and-mouth disease virus $2 \mathrm{~A}$ ) sequence to mediate self-processing of a polyprotein (Halpin et al., 1999). In this way, the MPI and PCI polypeptides were fused into a single open reading frame using the FMVD 2A sequence (hereafter referred to as MPI-2A-PCI protein). The second strategy consisted the use of the nucleotide sequence encoding the processing site of the cry $1 \mathrm{~B}$ precursor protein to fuse the $\mathrm{mpi}$ - and $p c i$-coding sequences (hereafter referred to as MPI-C-PCI protein). In this respect, it is well known that the Cry proteins are synthesised as longer inactive precursor proteins which are proteolytically processed by trypsin-like proteinases in the insect gut to become an active toxin (Miranda et al., 2001). Knowing the functional importance of the C-terminal tail of $\mathrm{PCI}$ for inhibition of carboxypeptidase activities (Marino-Buslje et al., 2000), the pci gene was fused in frame and downstream of the mpi gene in the two strategies.

The two mpi-pci fusion genes were expressed in rice under the control of the mpi promoter (-1872/+197 promoter region). This promoter has been shown to be activated in vegetative tissues of transgenic rice in response to mechanical wounding (a plant response to insect feeding) and fungal infection (Breitler et al., 2001; Moreno et al., 2005). The components of the two plant expression vectors used for wound-inducible expression of each mpi-pci fusion gene in rice plants are shown in Figure $1 \mathrm{a}$.

Transgenic rice (O. sativa elite japonica cultivar Ariete) plants were generated by Agrobacterium-mediated transformation. Transgene integration in the rice genome was assessed by Southern blot hybridization, most of the plants showing 1 or 2 integration events (Figure S1). The wound-inducible expression of the mpi-pci gene in leaves of transgenic rice plants was confirmed through successive generations (T0 to T3 progeny plants) by Northern blot analysis (Figure $1 \mathrm{~b}$ shows the results obtained on the analysis of T2 homozygous plants). No accumulation of mpipci transcripts occurred in either wounded leaves from untransformed plants (Figure 1b, WT; see Methods S1 for wounding of plant leaves) or unwounded leaves of transgenic plants (results not shown). Finally, no visible pleiotropic effects on plant growth and development due to the expression of the mpi-pci fusion gene were observed (results not shown).

\section{Effect of transgenic expression of the mpi-pci fusion gene on insect growth}

To determine the effect of transgenic expression of a mpi-pci gene on the growth of $C$. suppressalis larvae, a series of feeding trials were carried out. In these experiments, five independent homozygous lines for each fusion gene ( $m p i-C-p c i$ and $m p i-2 A$ pci lines) and wild-type plants were assayed (Methods S1). These studies revealed a significant reduction in weight gain in the larvae fed on rice lines expressing one or another mpi-pci fusion gene when compared with larvae fed on wild-type plants (Figure 2a). After feeding for 14 days, the average weight of larvae fed on control plants was $38.8 \pm 3.1 \mathrm{mg}$. The weight of larvae fed on mpi-C-pci lines was, however, significantly lower than that of larvae fed on control plants, their mean weight ranging from 13.7 to $23.4 \mathrm{mg}$ depending on the line (percentage of weight reduction, $64.6 \%-39.6 \%$ ) (Figure $2 a$ ). Similarly, larvae fed on mpi-2A-pci plants grew significantly slower that those fed on control plants, these larvae reaching a mean weight of 22.1 to $32.5 \mathrm{mg}$ (percentage of weight reduction, 42.9\%-16.2\%) (Figure 2a). Representative images of larvae recovered at the completion of the 14-day experimental period are shown in Figure $2 \mathrm{~b}$. On the basis of the weight of larvae recovered in these feeding experiments, the larval population was distributed into different groups. Whereas larvae recovered from wild-type plants distributed along the various weight classes and reached the higher weight classes (>70 mg), most of the larvae recovered from mpi-pci lines remained in the lower weight classes, and none of the larvae recovered from these plants reached the 70 mg weight mark (Figure S2). Together, feeding trials established that larvae fed on rice plants expressing a mpi-pci gene under a wound-inducible regime showed an important reduction in their weight when compared with that of larvae fed on control plants. 
(a) Vector 1: pC1300::mpi prom::mpi-2A-pci::mpi ter

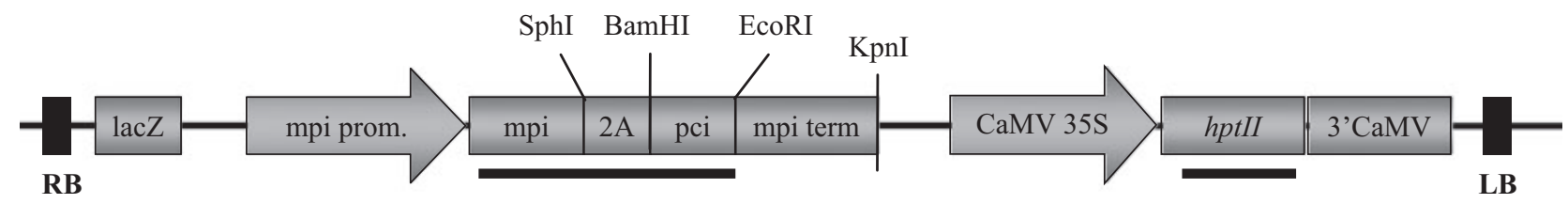

(b) Vector 2: pC1300::mpi prom::mpi-C-pci::mpi ter
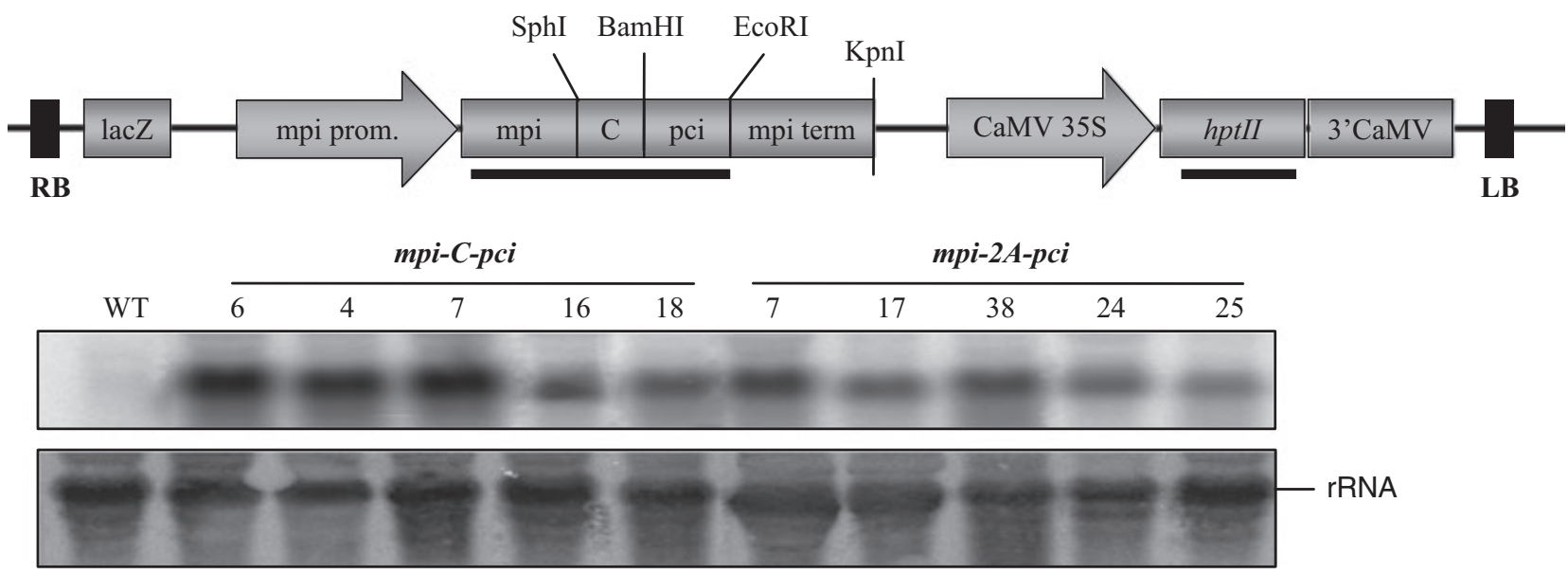

Figure 1 Expression of the mpi-pci fusion gene in transgenic rice plants. (a) Schematic representation of vectors used for rice transformation. Vector 1 contained the mpi-2A-pci fusion gene, whereas vector 2 contained the mpi-C-pci fusion gene. The wound-inducible mpi promoter was used to drive the expression of the mpi-pci fusion gene. The hptll gene encoding resistance to hygromycin served as the selectable marker in rice transformation The regions used as probe for genomic Southern blot analyses (Figure S1) are indicated with solid lines. (b) Northern blot analysis of total RNA isolated from wounded leaves of transgenic and control untransformed (WT, Ariete) rice plants. Representative lines transformed with the mpi-2A-pci or the mpi-Cpci fusion gene (T2 generation) are shown. Hybridization to the $18 \mathrm{~S}$ ribosomal RNA is shown in the lower panel as a control for loading.

\section{Wound-inducible expression of the mpi-pci fusion gene in rice confers insect resistance}

Knowing that rice plants expressing a mpi-pci fusion gene had a negative impact on larval growth, we assessed the performance of rice plants during infestation with $C$. suppressalis larvae under controlled greenhouse conditions. Three weeks after infestation, transgenic plants, either the mpi-C-pci or the mpi-2A-pci lines, showed normal development, whereas control plants were severely damaged or dead (Figure 3a). Furthermore, Northern blot analysis revealed important levels of mpi-pci transcript accumulation in leaves of all the transgenic lines used in these bioassays, this observation indicating that the mpi promoter directs high level of transgene expression in C. suppressalisinfested rice plants (Figure $3 \mathrm{~b}$ ). This is in agreement with the results previously reported on the mpi promoter activity in transgenic rice (Breitler et al., 2001, 2004; Vila et al., 2005).

It is well known that infestation by $C$. suppressalis leads to the formation of empty panicles, a condition commonly called 'white head'. Accordingly, we determined the percentage of white panicles in rice plants that have been infested with $C$. suppressalis larvae. For this, a subset of noninfested and C. suppressalisinfested transgenic and wild-type plants were allowed to continue growth and further analysed for production of white panicles (Figure 3c). Whereas the percentage of white panicles in wild-type plants was $20.5 \%$, the transgenic lines produced a percentage of white panicles ranging from $5.9 \%$ to $12.5 \%$ in the mpi-C-pci lines or from $7.7 \%$ to $13.6 \%$ in the mpi-2A-pci lines.
Collectively, results obtained in insect bioassays revealed that the expression of the mpi-pci fusion gene in rice plants confers protection against the lepidopteran insect C. suppressalis. In addition to prevent plant damage caused by $C$. suppressalis, rice plants expressing a mpi-pci gene produced less white panicles than wild-type plants.

\section{Analysis of transgene-derived protein products}

Immunoblot experiments were carried out to examine the accumulation of the transgene products in rice tissues in each strategy assayed in this work. For this, protein extracts were prepared from wounded leaves of wild-type and transgenic plants, both mpi-C-pci and mpi-2A-pci plants (homozygous T2 generation), and probed with an anti-MPI antibody raised against the pure MPI protein (Tamayo et al., 2000) (for details on Western blot analysis see Methods S1). As a control, protein extracts from wounded leaves of rice plants expressing the mpi gene under the control of its own promoter (Vila et al., 2005) were analysed. As expected, the MPI polypeptide accumulated in mpi rice plants (Figure 4a). Immunoblot analysis of protein extracts from mpi-C-pci lines also confirmed the accumulation of the fusion protein, which was absent in wild-type plants (Figure 4a). No immunological reactions occurred with the protein extracts from rice plants when the pre-immune serum was used (results not shown).

The expression strategy using the 2A sequence as the linker peptide aimed to produce the two individual proteinase inhibitor proteins, $\mathrm{MPI}$ and $\mathrm{PCl}$, from the hybrid protein in rice tissues. Immunoblot analysis of protein extracts obtained 
(a)

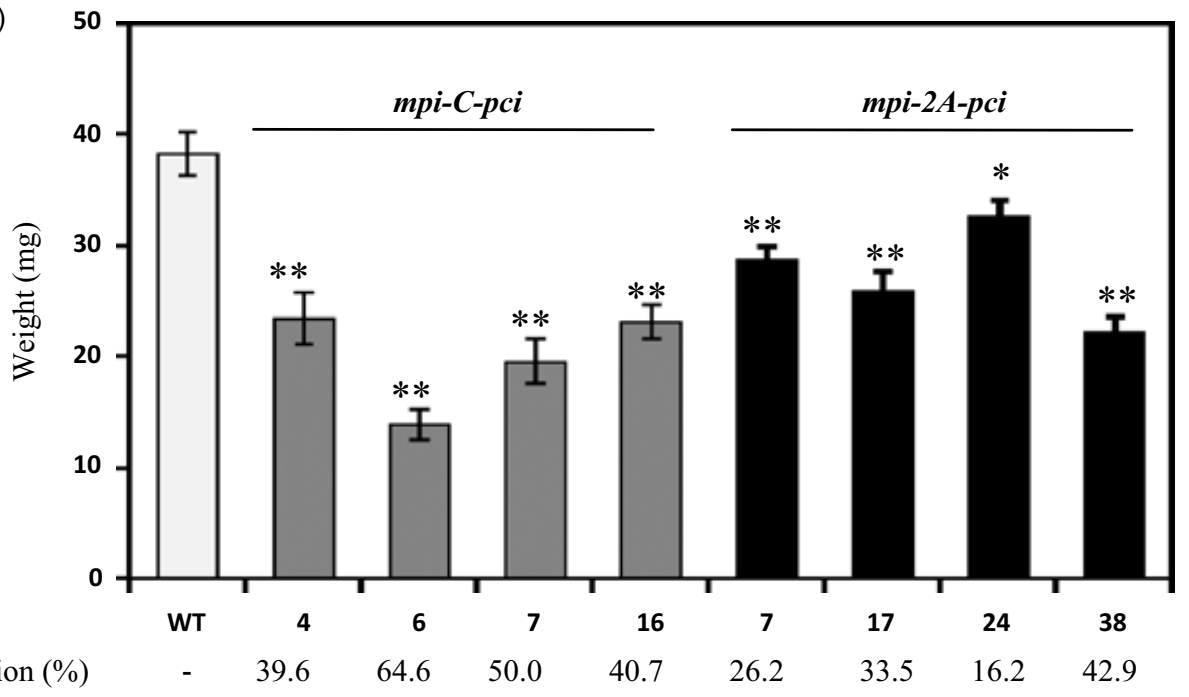

(b)
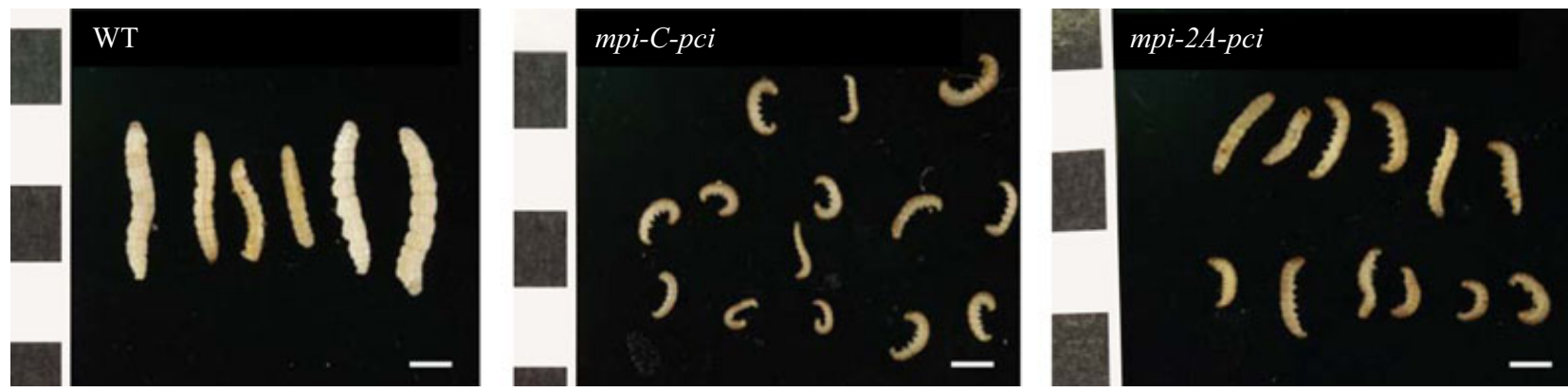

Figure 2 Insect feeding bioassays. (a) Growth of Chilo suppressalis fed on control wild-type (WT, white bar), mpi-C-pci (grey bars) and mpi-2A-pci (black bars) plants (homozygous T2 lines). Fourteen days after infestation with L2 C. suppressalis larvae, plants were dissected and larvae were recovered and individually weighted. The percentage of weight reduction in larvae fed on each transgenic line relative to larvae recovered from control plants is indicated at the bottom. Data sets were analysed for significant differences using Student's t-test $(* * P \leq 0.001 ; * P \leq 0.05)$. Error bars indicate the standard deviation of the error (SE). The distribution of the larval population according to their weight for each line is presented in Figure S2. (b) Representative C. suppressalis larvae recovered from wild-type (WT), mpi-C-pci (line 6) or mpi-2A-pci (line 38) rice plants. Bar $=0.5 \mathrm{~cm}$

from the mpi-2A-pci lines revealed accumulation of both, the fusion uncleaved MPI-2A-PCI protein and the MPI protein, indicating that the fusion protein was partially processed in the rice leaves (Figure 4a). The cleavage efficiency varied among the different transgenic lines. By comparing the band intensities with those of known amounts of MPI protein, the MPI content in mpi-2A-pci lines was estimated to be $0.10-0.25 \%$ of total soluble proteins (\% of MPI in total soluble protein) depending on the transgenic line. Longer exposure times of immunoblots containing protein extracts from mpi-2A-pci plants indicated that the MPI-2A-PCl polyprotein was properly cleaved as revealed by the absence of $\mathrm{MPI}$-containing truncated protein signals (Figure S3).

During the course of this work, the pure $\mathrm{PCl}$ protein was used to raise polyclonal antibodies in either rabbits or egg yolk which were then used for immunoblot analysis of protein extracts from rice leaves. Unfortunately, these analyses did not allowed us to detect the $\mathrm{PCI}$ polypeptide in the same protein extracts in which MPI was detected. Detection problems arising from inefficient extraction of $\mathrm{PCl}$ from plant tissues, or low antigenicity of the $\mathrm{PCl}$ polypeptide, might explain these results. In favour of the low antigenicity of $\mathrm{PCl}$, pure $\mathrm{PCl}$ polypeptide was undetectable by Western blot analysis (data not shown).
Failure to detect $\mathrm{PCl}$ in rice protein extracts prompted us to examine $\mathrm{PCl}$ activity in leaves of $\mathrm{mpi}$-pci rice plants. For this, total protein extracts were prepared from wounded leaves of either mpi-C-pci or mpi-2A-pci plants which were then subjected to affinity chromatography on immobilized carboxypeptidase $A$ (CPA). As controls, pure $\mathrm{PCl}$ protein as well as protein extracts from leaves of rice plants constitutively expressing pci (Quilis et al., 2007) was subjected to affinity chromatography. In all the cases, the eluted fractions were assayed for their ability to inhibit bovine CPA activity. When pure $\mathrm{PCl}$ protein or protein extracts from pci rice plants were analysed, the inhibitory activity of $\mathrm{PCI}$ against CPA was recorded in fractions eluted at $\mathrm{pH} 11.0$, but not in protein fractions eluted at $\mathrm{pH} 9.0$ (Figure 4b, upper panels). Equally, when protein extracts from mpi-C-pci or mpi-2A-pci plants were subjected to affinity chromatography, the protein fractions eluted at $\mathrm{pH} 11.0$ showed inhibitory activity against CPA (Figure $4 \mathrm{~b}$, lower panels). A certain level of CPA inhibition in the first fraction eluted at $\mathrm{pH} 9.0$ (mpi-C-pci plants) was also detected, pointing to a weaker interaction of the fusion protein with CPA during affinity chromatography relative to that of the $\mathrm{PCl}$ alone.

Together, these results suggest that the two mpi-pci fusion genes are efficiently transcribed and translated to produce a MPI- 
(a)

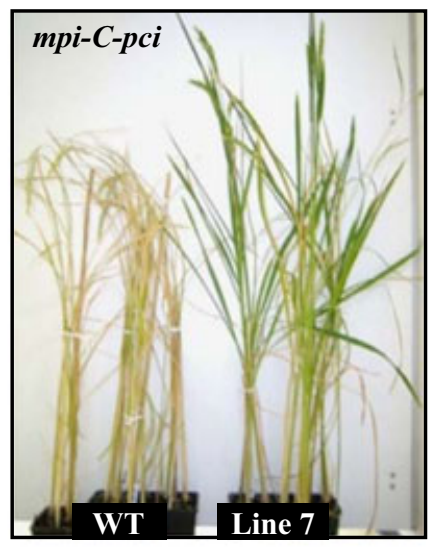

(b)

mpi-C-pci

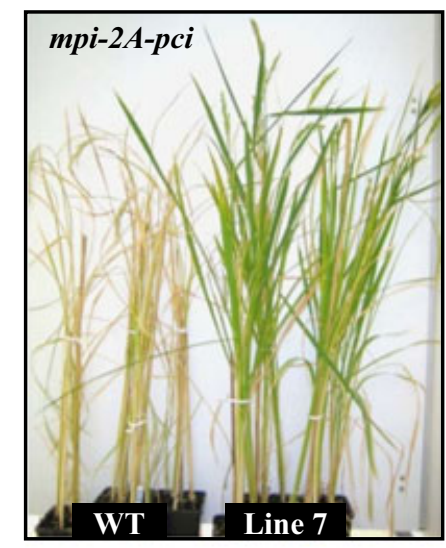

mpi-2A-pci
Figure 3 Resistance to $C$. suppressalis of rice plants expressing a mpi-pci fusion gene. (a) Phenotype of wild-type (WT) and mpi-pci plants that had been infested with $C$. suppressalis larvae. Bioassays for insect resistance were performed with mpi-C-pci (lines 4, 6, 7, 16 and 18) and mpi2A-pci (lines 7, 17, 24, 25 and 38) plants. Results for the mpi-C-pci line 7 and the mpi-2A-pci line 7 are shown (similar results were obtained for the other lines here assayed for each gene). (b) Northern blot analysis of RNAs obtained from mpi-C-pci or mpi-2A-pci rice plants used in bioassay experiments. Leaves from at least 6 plants for each line were pooled and analysed by Northern blot (20 $\mu \mathrm{g}$ of total RNA each sample). Ethidium bromide staining served as loading controls. (c) Percentage of white panicles in rice plants infested with C. suppressalis larvae relative to the total panicles obtained for each line. Data shown are from one of two experiments that produced similar results.
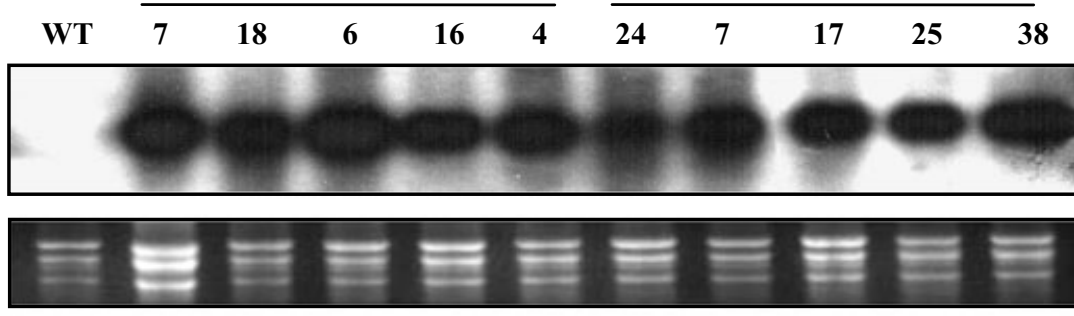

(c)

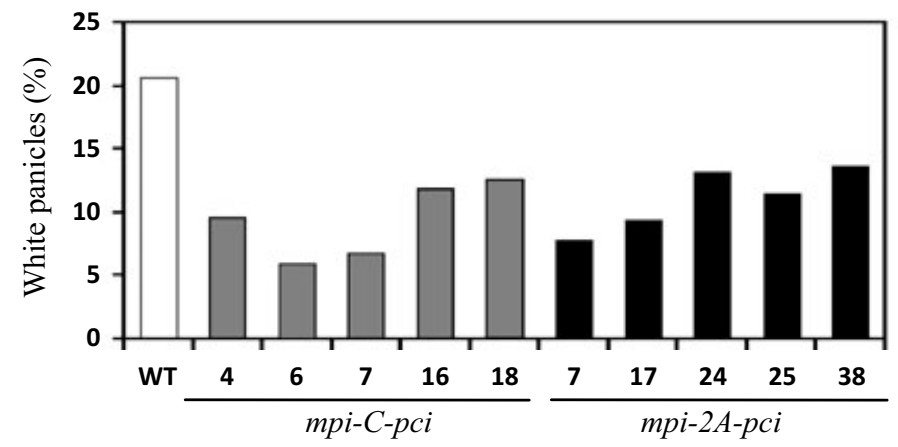

$\mathrm{PCl}$ fusion protein when fused by either the FMDV $2 \mathrm{~A}$ sequence or the processing sequence of the Cry1B precursor protein. Concerning the mpi-2A-pci rice plants, processing of the fusion protein and accumulation of the MPI polypeptide in rice tissues was demonstrated. Limitations for immunodetection of $\mathrm{PCl}$ by Western blot analysis hampered detection of the $\mathrm{PCI}$ polypeptide produced by cleavage of the MPI- $2 \mathrm{~A}-\mathrm{PCI}$ fusion protein in rice tissues. Even though the accumulation of the $\mathrm{PCl}$ polypeptide plants could not be clarified in this work, we have shown that protein extracts from mpi-C-pci plants exhibited inhibitory activity against CPA, supporting that the $\mathrm{PCI}$ domain of the fusion protein is active and effective for inhibition of carboxypeptidase activity.

\section{Resistance of mpi-pci rice plants to infection by the rice} blast fungus Magnaporthe oryzae

In this work, the functional relevance of transgenic expression of the mpi-pci fusion gene in terms of resistance to infection by $M$. oryzae was examined. Results previously reported by our group indicated that rice plants constitutively expressing the pci gene exhibit enhanced resistance to infection by this fungus (Quilis et al., 2007). Accordingly, resistance to fungal infection of mpi-pci rice plants was determined and compared to that of pci rice plants (Methods S1). For this, leaves of the transgenic plants were locally inoculated with spores from a gfp-expressing $M$. oryzae isolate as previously described (Campos-Soriano and San Segundo, 2009). Differences in the degree of disease symptoms caused by M. oryzae between mpi-pci lines and wild-type plants were clearly observed. At 3 days after inoculation, small lesions were generally observed in leaves of the mpi-pci and pci lines, whereas the leaves from control plants were visibly damaged under the same experimental conditions (Figure 5a). Fluorescence microscopy revealed the presence of a fluorescent hyphae growing on the leaf surface of wild-type plants (Figure 5a).

Finally, image analysis was used to determine the percentage of leaf area affected by blast lesions in mpi-pci and pci plants at 6 days postinoculation. In agreement with the visual inspection, the inoculated leaves from mpi-pci and pci transgenic lines exhibited a lower percentage of diseased area relative to inoculated leaves from wild-type plants (Figure 5b). Generally, the rice lines expressing the $m p i-C-p c i$ gene exhibited relatively reduced blast disease symptoms compared to the transgenic lines expressing either the mpi-2A-pci gene or the pci gene alone.

Together, disease resistance assays demonstrated that expression of the mpi-pci fusion gene in rice, either the mpi-2A-pci or the mpi-C-pci gene, confers enhanced resistance against the rice blast fungus $M$. oryzae. Furthermore, these results indicated that the 
(a)

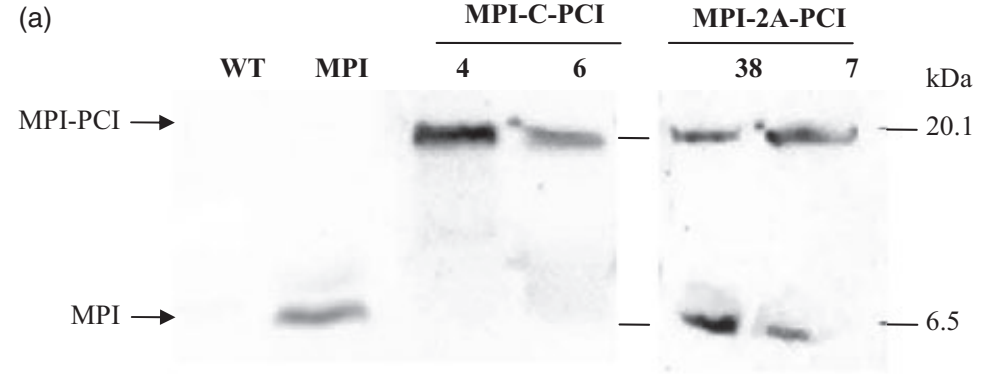

(b)

$$
\text { PCI }
$$

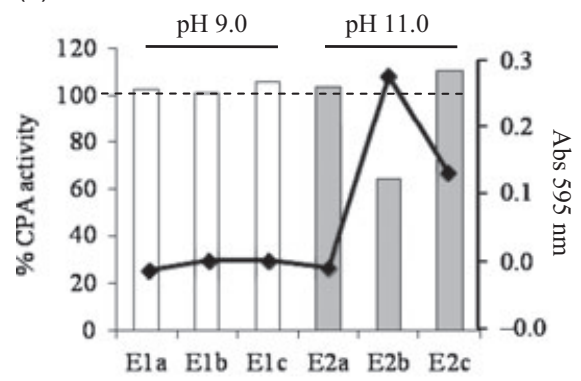

MPI-C-PCI

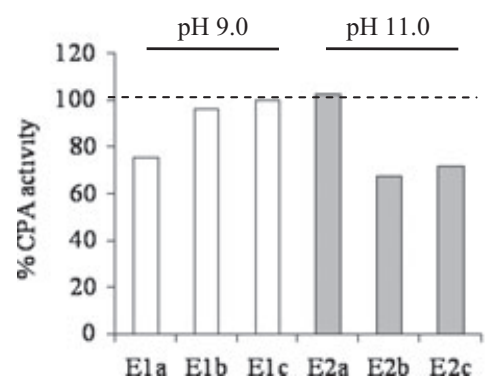

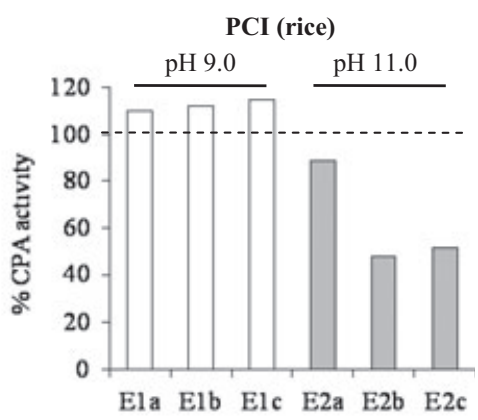

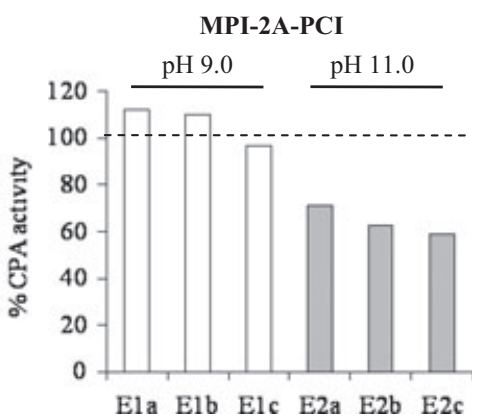

Figure 4 Protein analysis of transgenic plants expressing a mpi-pci fusion gene. (a)

Immunodetection of MPI in total protein extracts obtained from leaves of wild-type (WT) plants and plants harbouring the mpi gene (Vila et al., 2005), the mpi-C-pci (lines 4 and 6) or the mpi-2A-pci (lines 38 and 7). In all the cases, transgene expression was driven by the wound-inducible $m p i$ promoter. Leaves were mechanically wounded and harvested at $16 \mathrm{~h}$ after wounding. Protein extracts ( $40 \mu \mathrm{g}$ each extract) were separated by 15\% SDS-PAGE and probed with an anti-MPI antibody (Tamayo et al., 2000). (b) Affinity chromatography on immobilized CPA of pure PCI (Sigma) and protein extracts from rice plants constitutively expressing pci (line 7.34), mpi-C-pci plants (line 7) and mpi-2A-pci (line 7) plants. Eluted fractions were analysed for inhibition of bovine CPA activity using AAFP as the substrate (Quilis et al., 2007). Lanes E1a to E1c, fractions eluted using buffer at pH 9.0. Lanes E2a to E2c, fractions eluted using buffer at $\mathrm{pH}$ 11.0. In each sample, CPA activity is referred to the activity of the enzyme in the corresponding buffer (considered as 100\%).
MPI-C-PCI fusion protein might be effective for inhibition of fungal proteinases (as it was previously demonstrated for $\mathrm{PCl}$; Quilis et al., 2007). Knowing that the fusion protein is partially processed in rice leaves of mpi-2A-pci (see Figure 4a), the contribution to the phenotype of disease resistance that is observed in these plants might be due to the activity of the MPI-2A-PCI protein and the PCI protein originating from the fusion protein.

\section{Discussion}

In this work, we show that the expression of a fusion gene encoding two proteinase inhibitors, namely the MPI and PCI inhibitors, in transgenic rice provides dual resistance against insect attack and pathogen infection. The protective effect of the fusion gene was achieved when using either the FMDV2A linker sequence or the processing region of the Cry1B precursor protein to fuse the two proteinase inhibitor polypeptides. Although evidence exists in the literature on the insecticidal properties of plant Pls, very limited information is currently available about a dual protective effect against insects and pathogens in transgenic crops expressing plant Pls. Only, the simultaneous expression of a trypsin inhibitor (sporamin) gene and a phytocystatin gene in transgenic tobacco plants was reported to be effective for resistance against insect and pathogen attack (Senthilkumar et al., 2010). More recently, the UNUSUAL SERINE PROTEASE INHIBITOR (UPI) gene was reported to function in resistance to necrotrophic fungi and insect herbivory in Arabidopsis plants (Laluk and Mengiste, 2011).

The transgenic rice expressing a mpi-pci fusion gene under the control of the mpi promoter showed normal growth and development. In other studies, the constitutive expression of proteinase inhibitor genes in transgenic plants has been shown to have a negative impact on plant development (Zavala et al., 2004). Clearly, the use of a wound- and pathogen-inducible mpi promoter (instead of a constitutive promoter) minimizes the possibility of alterations in fitness of the transgenic plants while driving levels of transgene expression sufficient to confer protection to the plant. An additional advantage of using the mpi promoter is that this promoter is not active in pollen, seed embryo or endosperm of rice plants, thus reducing food and environmental concerns (Breitler et al., 2001, 2004). Together, the results here presented suggest that the mpi promoter can be considered a good candidate to drive conditional expression of insecticidal and antifungal genes in transgenic rice.

Insect feeding experiments revealed that the weight gain of C. suppressalis larvae fed on mpi-pci plants was reduced compared to that of larvae fed on wild-type plants. Based on the inhibitory properties of MPI and $\mathrm{PCI}$ (MPI inhibits insect elastase and chymotrypsin activities, whereas $\mathrm{PCl}$ inhibits insect carboxypeptidases), a broad spectrum of insect digestive proteinases is expected to be affected by the coordinated and complementary activity of the two inhibitors. This fact might well explain 
(a)

Figure 5 Resistance of rice plants expressing the mpi-pci gene to $M$. oryzae. (a) Leaves from transgenic rice lines harbouring the $m p i-C-p c i$, mpi-2A-pci, pci or wild-type (WT) plants were inoculated with spores of the $g f p$-expressing M. oryzae isolate $\left(10^{6}\right.$ spores $\left./ \mathrm{mL}\right)$ as previously described (Campos-Soriano et al., 2013). Disease symptoms were observed by light and fluorescent microscopy at 3 days after inoculation. Representative results from one of three experiments that produced similar results are shown. (b) Percentage of leaf area affected by blast lesions was determined using the image analysis software Assess v. 2.0 for plant disease quantification at 6 days after inoculation with M. oryzae spores. Data sets were analysed for significant differences using Student's $t$-test $(* P \leq 0.1)$. Results are shown as mean values $\pm \mathrm{SE}$.

the observed effectiveness of this strategy in conferring insect resistance. The mean weight reduction in larvae fed on rice plants expressing a fusion gene was $39.6 \%-64.6 \%$ and $16.2 \%-42.9 \%$ for the mpi-C-pci and mpi-2A-pci lines, respectively. In this respect, we previously reported that transgenic expression of the mpi gene alone (also under the control of its own promoter) results in a mean weight reduction in C. suppressalis larvae of $23.0 \%-28.6 \%$ depending on the line (Vila et al., 2005). Further studies are, however, needed to conclusively determine whether transgenic expression of the mpi-pci fusion gene is more effective for inhibition of larval growth than the expression of the mpi gene alone.

On the other hand, results previously reported by our group indicated that production of the $\mathrm{PCl}$ inhibitor alone in the rice plant might be counterproductive in terms of insect resistance, as the weight gain of C. suppressalis larvae fed on rice plants constitutively expressing the pci gene was significantly larger than that of larvae fed on wild-type plants (Quilis et al., 2007). It can then be hypothesized that the production of the two plant inhibitors, MPI and $\mathrm{PCl}$, compensates the negative effects that occur in C. suppressalis larvae fed on rice plants expressing the pci gene alone. If so, the simultaneous production of the $\mathrm{PCl}$ and MPI inhibitors in rice would counteract adaptive mechanisms in the complement of digestive proteinases and minimize the compensatory response of $C$. suppressalis larvae after ingestion of the $\mathrm{PCl}$ alone.

Concerning the mechanisms underlying insect resistance in mpi-pci rice plants, two scenarios can be envisaged. Firstly, there
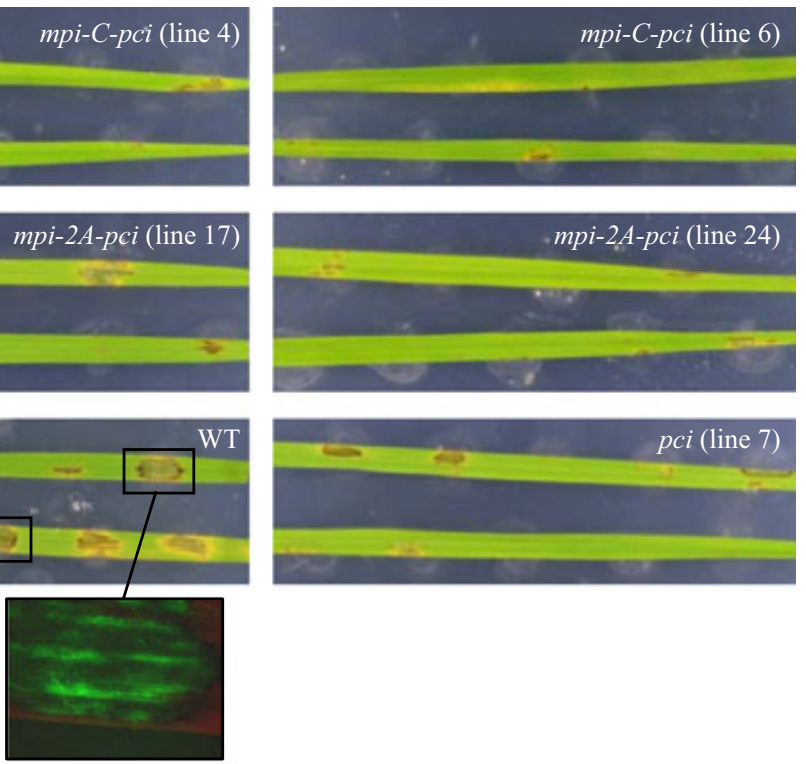

mpi-2A-pci (line 17)

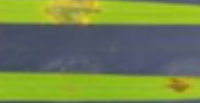

WT
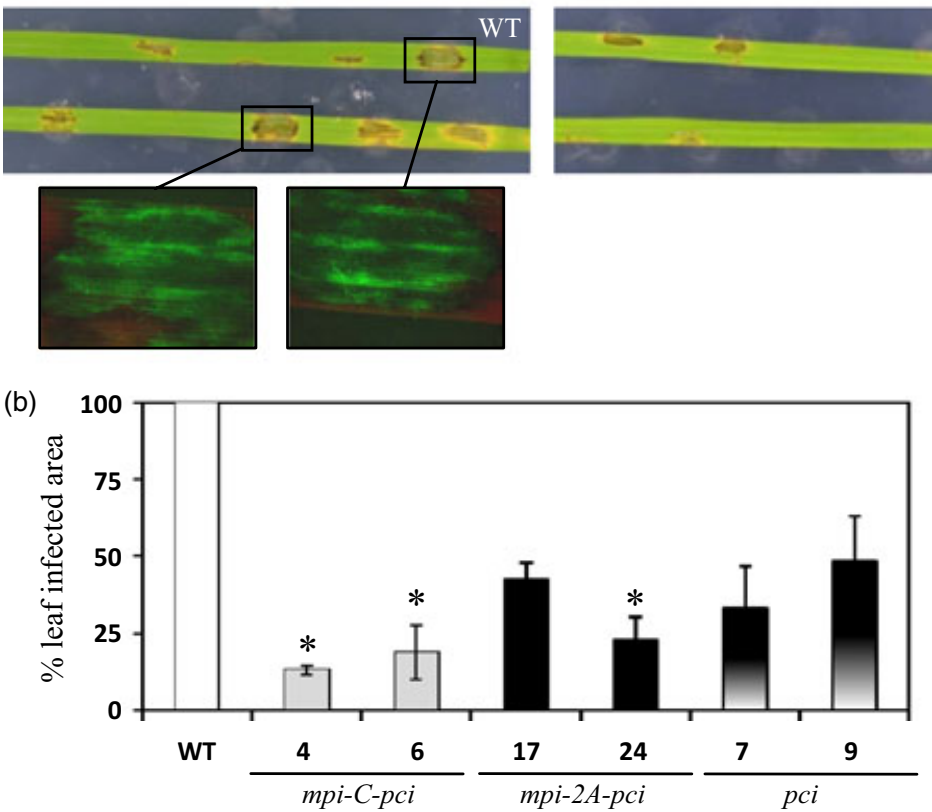

is the possibility that the MPI-PCI fusion protein acts as a multifunctional proteinase inhibitor. In this respect, efforts have been made to obtain multifunctional proteinase inhibitors exhibiting activity against multiple target proteinases (Schluter et al., 2010). Such approaches involve chimeric proteins that integrate complete or partial functionally significant regions of the inhibitor sequences with a broader inhibitory range against target proteinases (Brunelle et al., 2005; Inanaga et al., 2001; Outchkourov et al., 2004). Most of these studies, however, have been approached by in vitro inhibition assays on insect gut protein extracts or by insect feeding assays using artificial diets supplemented with isolated Pls. Secondly, the activity of the two partners originating from the fusion protein either in the rice tissue (mpi-2A-pci plants) or in the gut of $C$. suppressalis larvae (mpi-C-pci plants) would be responsible for the observed phenotype of resistance. In this respect, whereas the in vivo processing of the MPI-2A-PCl fusion protein in rice leaves has been confirmed in this work, the proteolytic processing of the fusion protein in the insect gut has not been demonstrated yet. Further analysis will aid in determining whether insect resistance in $\mathrm{mpi}$ C-pci and mpi-2A-pci rice plants is mediated by the MPI-PCI fusion protein acting as a multifunctional proteinase inhibitor or by the activity of the two independent inhibitors.

As for the $\mathrm{PCl}$ inhibitor, it is well known that the $\mathrm{C}$-tail residues of the $\mathrm{PCl}$ polypeptide are essential for enzyme inhibition (Marino-Buslje et al., 2000). There is then a possibility that the $\mathrm{PCl}$ protein, either alone or in the form of uncleaved MPI-PCI fusion protein, exerts its inhibitory activity against carboxypepti- 
dase activities. The observation that protein extracts from mpi-Cpci plants exhibit inhibitory properties against carboxypeptidase activity, together with the observed phenotype of blast disease resistance in these plants, favours the possibility that the $\mathrm{PCI}$ domain of the MPI-C-PCI fusion protein is functional and active for inhibition of target carboxypeptidases. Then, fusing the $\mathrm{PCI}$ polypeptide to the $\mathrm{C}$-terminus region of proteins of interest in the form of polyproteins might then be a suitable strategy to obtain insect and/or pathogen resistance in transgenic plants.

Moreover, we show that expression of a mpi-pci fusion gene in rice does not confer levels of protection against $M$. oryzae significantly higher than those occurring in the rice plants expressing the pci gene alone. Contrary to the pci rice plants, the rice plants expressing the mpi gene alone do not show resistance to infection by the rice blast fungus $M$. oryzae (results not shown). From these observations, it can be reasoned that the protective effect that is observed in mpi-C-pci and mpi-2A-pci plants results from the antifungal properties of $\mathrm{PCI}$ (Quilis et al., 2007). If so, the two inhibitors would not act in a synergistic manner in conferring resistance to $M$. oryzae infection in transgenic rice.

Concerning the strategy based on the use of the FMVD2A sequence, it should be here mentioned that this strategy has been used for co-expression of different genes in transgenic plants. Some examples are the expression of reporter genes in tobacco (de Felipe et al., 2006; Halpin et al., 1999; Ma and Mitra, 2002), the production of lignocelluloses degradation enzymes or selfcleaving antimicrobial polyproteins in tobacco or Arabidopsis plants (François et al., 2004; Lee et al., 2012), or coexpression of carotenoid or tyramine derivatives biosynthetic enzymes in the rice endosperm (Park et al., 2009; Ralley et al., 2004). However, when using the FMDV2A sequence to fuse two antimicrobial proteins (snaking and defensin) in the form of a hybrid protein, no cleavage products were detected in tobacco and potato plants (Kovalskaya et al., 2011). Our in vivo experiments demonstrated a clear phenotype of resistance to insect attack and pathogen infection in the mpi-2A-pci rice plants, despite the lack of complete processing of the in planta-produced fusion protein. At present, the contribution of the fusion MPI-2A-PCI protein and the individual proteins derived from the fusion protein (MPI and $\mathrm{PCI}$ ) to the observed phenotype of resistance against $C$. suppressalis remains unknown. Altogether, these findings support the need of addressing on a case-by-case basis the evaluation of the FMVD2A sequence as a linker peptide to fuse proteins of interest in a particular plant species.

An important advantage of the strategy assayed in this work is that a single transformation event can lead to the production of plant Pls with different modes of action using only a single promoter, thus avoiding the risk of homology-based silencing, transgene segregation along successive generation or the need of different promoters or selectable markers (as it occurs in other strategies for co-expression of multiple transgenes). Clearly, different approaches can be used for the simultaneous expression of insecticidal and/or antifungal genes in plants, each one having its own advantages and drawbacks to be adopted in crop biotechnology. For instance, the integration of multiple transgenes in a single plant can be achieved by either crossing individual transformants, by sequential transformation, and by co-transformation either with a single plasmid equipped with multicassettes or with multiple plasmids can be approached. Such strategies are, however, time-consuming and require the use of different selectable marker genes. For single-plasmid co-transformation, the main technical limitation is the difficulty to assemble complex plasmids with multiple gene cassettes, whereas the success of co-transformation with multiple plasmids depends on the frequency by which independent transgenes are integrated into the host genome (transgenes can also segregate in successive generations). Expression of multiple genes in a single transgenic plant also requires the use of different promoters to avoid homology-dependent transgene silencing. Here, it is worthy to mention that there are few reports on insect resistance based on the co-expression of multiple proteinase inhibitors in transgenic plants. Gene pyramiding for insect resistance using plant Pls has been reported by crossing transgenic lines expressing individual genes or using a plant transformation vector that harbours a double expression cassette (Abdeen et al., 2005; Dunse et al., 2010).

Due to the focus on agricultural importance, expression of genes encoding multiple insecticidal factors appears to be critical for long-term control of insect pests. Knowing that the two components of the MPI-PCl fusion protein are effective for inhibition of insect proteinases with different mechanisms of action (quimotrypsin and elastase by MPI, carboxypeptidases by $\mathrm{PCI}$ ), the simultaneous expression of these plant proteinase inhibitors is expected to maintain its insecticidal properties longer than inhibitors acting on proteinases with a single mechanistic mode of action by hindering insect adaptation to proteinase inhibitor ingestion. The use of mpi-pci fusion genes, either alone or adjunct to Bt, can thus be considered as a promising strategy for the long-term protection of rice plants against the striped stem borer C. suppressalis. As an additional advantage, the transgenic expression of the mpi-pci fusion gene in rice confers protection against the rice blast fungus $M$. oryzae. This fungus is the causal agent of the rice blast disease, one the most devastating fungal disease of cultivated rice worldwide (Talbot, 2003). Currently, the control of the rice blast disease depends on the use of chemically synthesized fungicides. Along with this, the mpi-pci rice plants represent an alternative to the economically costly and environmentally undesirable chemical control of the rice blast disease. The ultimate goal of insect and pathogen resistance of the rice plants expressing a mpi-pci fusion gene needs now to be confirmed by further evaluation in the field.

\section{Experimental procedures}

\section{Construction of plant expression vectors and rice transformation}

Two vectors containing a mpi-pci fusion gene were prepared in this work and used for rice transformation (Figure 1a, Table S1). In both cases, the coding sequence of the pci gene was fused in frame and downstream of the mpi gene through either the FMVD2A linker sequence (mpi-2A-pci gene) or the cleavage site of the Cry1B precursor protein (mpi-C-pci gene). Expression of each fusion gene is driven by the mpi promoter and terminator regions (Moreno et al., 2005). Transformation was carried out using the Mediterranean elite japonica rice (Oryza sativa L.) cultivar Ariete. Details on preparation of expression vectors and production of transgenic rice plants by Agrobacterium-mediated transformation of embryogenic calli derived from mature embryos are indicated in Supporting Information. To plants were transferred to a containment glasshouse and examined for transgene expression and integration. Homozygous transgenic lines were identified in the $T_{2}$ generation. All rice plants were grown at $27 \pm 2{ }^{\circ} \mathrm{C}$ under 18-h/6-h light/dark photoperiod. 


\section{RNA and DNA analyses}

Total RNA was isolated from the leaves of transgenic and control plants using Trizol reagent according to the manufacturer's instructions (Invitrogen, Carlsbad, CA, USA). For Northern blot analysis, RNAs were subjected to formaldehyde-containing agarose gel electrophoresis and transferred to Hybond-N membranes (Amersham Biosciences, Piscataway, NJ). A DNA fragment coding for the specific mpi-pci fusion gene (mpi-2A-pci or mpi-C-pci) was used as a probe. All probes were gel-purified and radioactively labelled by random priming, according to the manufacturer's protocol (Roche Diagnostics, Mannheim, Germany). Hybridizations were conducted at $42{ }^{\circ} \mathrm{C}$ in $40 \%$ formamide in the hybridization solution.

Genomic DNA was extracted using MATAB (mixed alkyltrimethylammonium bromide) as the extraction buffer (Coca et al., 2004). For Southern blot analysis, genomic DNA was digested with $\mathrm{Kpnl}$ restriction enzyme, electrophoresed on $0.8 \%$ agarose gels, transferred onto nylon membranes (Hybond-N, Amersham, UK) and hybridized to DNA probes ${ }^{32} \mathrm{P}$-labelled by random priming (Roche Diagnostics $\mathrm{GmbH}$, Mannheim, Germany). Hybridizations were carried out at $65^{\circ} \mathrm{C}$ (Coca et al., 2004).

\section{Preparation of protein extracts and immunoblotting}

Protein extracts were prepared from wounded leaves of transgenic and nontransformed plants. Plant material was ground with liquid nitrogen, and total proteins were extracted using $50 \mathrm{~mm}$ Tris- $\mathrm{HCl}, \mathrm{pH} 8,1 \mathrm{~mm}$ EDTA, 5\% glycerol, $1 \mathrm{~mm}$ dithiothreitol and $0.1 \%(\mathrm{v} / \mathrm{v})$ Triton $\mathrm{X}-100$ as the extraction buffer. Extractions were carried out at $4{ }^{\circ} \mathrm{C}$ for $2 \mathrm{~h}$ with continuous slow stirring. Protein quantification was performed using Bio-Rad protein assay reagents (Bio-Rad, Madrid, Spain). Immunoblots were prepared as indicated in Supporting Information. To determine the level of MPI accumulation in total protein extracts from leaves of $m p i-2 A$ pci plants, the MPI protein was expressed in Escherichia coli (Tamayo et al., 2000), and different dilutions of MPI protein were applied to each blot as standards. The MPI levels were measured from digitalized images of the blots (Quantity One Program from Bio-Rad).

\section{Carboxypeptidase A affinity chromatography and activity assays}

Total protein extracts from transgenic plants were fractionated by affinity chromatography using a column of immobilized bovine CPA (CPA-agarose, Sigma, St Louis, MO, USA). The column was washed successively with $2.5 \mathrm{~mL}$ of $10 \mathrm{~mm}$ Tris- $\mathrm{HCl}, \mathrm{pH} 7.5$, $0.15 \mathrm{M} \mathrm{NaCl} ; 50 \mathrm{~mm}$ Tris- $\mathrm{HCl}, \mathrm{pH}$ 9.0; and $50 \mathrm{~mm} \mathrm{Na} \mathrm{HPO}_{4}, \mathrm{pH}$ 11.0. Eluted fractions $(0.5 \mathrm{~mL})$ were collected with each buffer and neutralized to $\mathrm{pH} 7.5$.

To determine the inhibitory activity of eluted fractions, $95 \mu \mathrm{L}$ of each fraction was pre-incubated with $5 \mu \mathrm{L}$ of $0.5 \mu \mathrm{m}$ bovine CPA (Sigma) for 10 min on ice. Then, CPA activity was assayed using $\mathrm{N}$-(4-methoxyphenyl-azoformyl)-L-phenylalanine (AAFP, Bachem, Bubendorf, Switzerland) at a final concentration of $0.05 \mathrm{~mm}$ (Quilis et al., 2007). The final volume of $200 \mu \mathrm{L}$ was added to a 96-well plates, and CPA activity was determined by decreasing absorbance at $350 \mathrm{~nm}$ over time. As positive control of inhibition, CPA activity was determined using different concentrations of commercial PCI (Sigma). For each fraction, the relative CPA activity was calculated as comparison of the CPA activity after addition of the corresponding elution buffer.

\section{Insect bioassays of transgenic plants}

Larvae of C. suppressalis were obtained from rice fields in the Camargue (France) and Delta del Ebro (Spain). Feeding assays with C. suppressalis larvae were performed as described by Vila and co-workers (Vila et al., 2005). Further experimental details can be found in Supporting Information. Resistance of transgenic rice plants to $C$. suppressalis was performed as described above, and plant symptoms (white panicles) were recorded after 21 days postinfestation.

\section{Blast resistance assays}

Resistance of mpi-pci and pci rice plants to infection by the rice blast fungus $M$. oryzae was carried out using the detached leaf assay (Coca et al., 2004) using a gfp-expressing $M$. oryzae isolate (gfp-PR9) (Campos-Soriano and San Segundo, 2009; CamposSoriano et al., 2013). Further experimental details can be found in Supporting Information.

\section{Acknowledgements}

BLG was a researcher from the Ministerio de Ciencia e Innovación (MICINN), Spain ('Ramon y Cajal'). We are grateful to M. Coca (CRAG, Spain) for her collaboration in this work and M. Royer (CIRAD, BGPI unit, France) for providing us with the $C$ region of the Cry1B sequence synthesized in the framework of an UNDPfunded project. We are also grateful to C. Halpin, Dundee University, UK) for providing us with the pGUS2AGFP plasmid). This work was supported by grants BIO2003-04936-C02-01/ BIO2012-32838 to B.S.S and AGL2010-16847 to B.L-G from Ministerio de Economía y Competitividad (MINECO). We also thank the Consolider-Ingenio CSD2007-00036 to CRAG and the Generalitat de Catalunya (Xarxa de Referencia en Biotecnología and SGR 09626) for substantial support.

\section{References}

Abdeen, A., Virgós, A., Olivella, E., Villanueva, J., Avilés, X., Gabarra, R. and Prat, S. (2005) Multiple insect resistance in transgenic tomato plants over-expressing two families of plant proteinase inhibitors. Plant Mol. Biol. 57, 189-202.

Bayés, A., Sonnenschein, A., Daura, X., Vendrell, J. and Avilés, F.X. (2003) Procarboxypeptidase $A$ from the insect pest Helicoverpa armigera and its derived enzyme. Eur. J. Biochem. 270, 3026-3035.

Breitler, J.C., Cordero, M.J., Royer, M., Meynard, D., San Segundo, B. and Guiderdoni, E. (2001) The $-689 /+197$ region of the maize protease inhibitor gene directs high level, wound-inducible expression of the cry $1 B$ gene which protects transgenic rice plants from stemborer attack. Mol. Breeding 7, 259-274.

Breitler, J.C., Vassal, J.M., Catala, M.M., Meynard, D., Marfà, V., Melé, E., Royer, M., Murillo, I., San Segundo, B., Guiderdoni, E. and Messeguer, J. (2004) Bt rice harbouring cry genes controlled by a constitutive or wound-inducible promoter: protection and transgene expression under Mediterranean field conditions. Plant Biotechnol. J. 2, 417-430.

Broadway, R.M. (1996) Dietary proteinase inhibitors alter complement of midgut proteases. Arch. Insect Biochem. Physiol. 32, 39-53.

Broadway, R.M. (1997) Dietary regulation of serine proteinases that are resistant to serine proteinase inhibitors. J. Insect Physiol. 43, 855-874.

Brunelle, F., Girard, C., Cloutier, C. and Michaud, D. (2005) A hybrid, broad-spectrum inhibitor of Colorado potato beetle aspartate and cysteine digestive proteinases. Arch. Insect Biochem. Physiol. 60, 20-31.

Campos-Soriano, L. and San Segundo, B. (2009) Assessment of blast disease resistance in transgenic $P R m s$ rice using a gfp-expressing Magnaporthe oryzae strain. Plant. Pathol. 58, 677-689. 
Campos-Soriano, L., Valè, G., Lupotto, E. and San Segundo, B. (2013) Investigation of rice blast development in susceptible and resistant rice cultivars using a gfp-expressing Magnaporthe oryzae isolate. Plant. Pathol. 62, 1030-1037.

Cloutier, C., Jean, C., Fournier, M., Yelle, S. and Michaud, D. (2000) Adult Colorado potato beetles, Leptinotarsa decemlineata compensate for nutritional stress on oryzacystatin I-transgenic potato plants by hypertrophic behavior and over-production of insensitive proteases. Arch. Insect Biochem. Physiol. 44, 69-81.

Coca, M., Bortolotti, C., Rufat, M., Peñas, G., Eritja, R., Tharreau, D., Martínez del Pozo, A., Messeguer, J. and San Segundo, B. (2004) Transgenic rice plants expressing the antifungal AFP protein from Aspergillus giganteus show enhanced resistance to the rice blast fungus Magnaporthe grisea. Plant Mol. Biol. 54, 245-259.

Cordero, M.J., Raventós, D. and San Segundo, B. (1994) Expression of a maize proteinase-inhibitor gene is induced in response to wounding and fungal infection: systemic wound-response of a monocot gene. Plant J. $\mathbf{6}$, 141-150.

Dale, D. (1994) Insect pests of the rice plant - Their biology and ecology. In Biology and Management of Rice Insects (Heinrichs, E.A., ed), pp. 363-485. Los Baños, Philippines: IRRI.

De Leo, F., Bonadé-Bottino, M.A., Ceci, L.R., Gallerani, R. and Jouanin, L. (1998) Opposite effects on Spodoptera littoralis larvae of high expression level of a trypsin proteinase inhibitor in transgenic plants. Plant Physiol. 118, 9971004

Duan, X., Li, X., Xue, Q., Abo-El-Saad, M., Xu, D. and Wu, R. (1996) Transgenic rice plants harboring an introduced potato proteinase inhibitor II gene are insect resistant. Nat. Biotechnol. 14, 494-498.

Dunse, K.M., Stevens, J.A., Lay, F.T., Gaspar, Y.M., Heath, R.L. and Anderson, M.A. (2010) Coexpression of potato type I and II proteinase inhibitors gives cotton plants protection against insect damage in the field. Proc. Natl Acad. Sci. USA 107, 15011-15015

de Felipe, P., Luke, G.A., Hughes, L.E., Gani, D., Halpin, C. and Ryan, M.D. (2006) E unum pluribus: multiple proteins from a self-processing polyprotein Trends Biotechnol. 24, 68-75.

François, I.E.J.A., Van Hemelrijck, W., Aerts, A.M., Wouters, P.F.J., Proost, P., Broekaert, W.F. and Cammue, B.P.A. (2004) Processing in Arabidopsis thaliana of a heterologous polyprotein resulting in differential targeting of the individual plant defensins. Plant Sci. 166, 113-121.

Gatehouse, J.A. (2002) Plant resistance towards insect herbivores: a dynamic interaction. New Phytol. 156, 145-169.

Girard, C., Le Métayer, M., Bonadé-Bottino, M., Pham-Delegue, M.H. and Jouanin, L. (1998a) High level of resistance to proteinase inhibitors may be conferred by proteolytic cleavage in beetle larvae. Insect Biochem. Mol. Biol. 28, 229-237.

Girard, C., Le Métayer, M., Zaccomer, B., Bartlet, E., Williams, I., Bonadé-Bottino, M., Pham-Delegue, M.-H. and Jouanin, L. (1998b) Growth stimulation of beetle larvae reared on a transgenic oilseed rape expressing a cysteine proteinase inhibitor. J. Insect Physiol. 44, 263-270.

Giri, A.P., Harsulkar, A.M., Deshpande, V.V., Sainani, M.N., Gupta, V.S. and Ranjekar, P.K. (1998) Chickpea defensive proteinase inhibitors can be inactivated by podborer gut proteinases. Plant Physiol. 116, 393-401.

Graham, J.S. and Ryan, C.A. (1981) Accumulation of a metallo-carboxypeptidase inhibitor in leaves of wounded potato plants. Biochem. Biophys. Res. Commun. 101, 1164-1170.

Halpin, C., Cooke, S.E., Barakate, A., El Amrani, A. and Ryan, M.D. (1999) Self-processing 2A-polyproteins - a system for co-ordinate expression of multiple proteins in transgenic plants. Plant J. 17, 453-459.

Haq, S.K., Atif, S.M. and Khan, R.H. (2004) Protein proteinase inhibitor genes in combat against insects, pests, and pathogens: natural and engineered phytoprotection. Arch. Biochem. Biophys. 431, 145-159.

Hilder, V.A., Gatehouse, A.M.R., Sheerman, S.E., Barker, R.F. and Boulter, D. (1987) A novel mechanism of insect resistance engineered into tobacco. Nature 330, 160-163.

Inanaga, H., Kobayasi, D., Kouzuma, Y., Aoki-Yasunaga, C., liyama, K. and Kimura, M. (2001) Protein engineering of novel proteinase inhibitors and their effects on the growth of Spodoptera exigua larvae. Biosci. Biotechnol. Biochem. 65, 2259-2264.
Johnson, R., Narvaez, J., An, G.H. and Ryan, C. (1989) Expression of proteinase inhibitors I and II in transgenic tobacco plants: effects on natural defense against Manduca sexta larvae. Proc. Natl Acad. Sci. USA 86, 9871-9875.

Jongsma, M.A., Bakker, P.L., Peters, J., Bosch, D. and Stiekema, W.J. (1995) Adaptation of Spodoptera exigua larvae to plant proteinase inhibitors by induction of gut proteinase activity insensitive to inhibition. Proc. Natl Acad. Sci. USA 92, 8041-8045

Kim, J.Y., Park, S.C., Hwang, I., Cheong, H., Nah, J.W., Hahm, K.S. and Park, Y. (2009) Protease inhibitors from plants with antimicrobial activity. Int. J. Mol. Sci. 10, 2860-2872.

Koiwa, H., Bressan, R.A. and Hasegawa, P.M. (1997) Regulation of protease inhibitors and plant defense. Trends Plant Sci. 2, 379-384.

Kovalskaya, N., Zhao, Y. and Hammond, R.W. (2011) Antibacterial and antifungal activity of a snakin-defensin hybrid protein expressed in tobacco and potato plants. Open Plant Sci. J. 5, 29-42.

Laluk, K. and Mengiste, T. (2011) The Arabidopsis extracellular UNUSUAL SERINE PROTEASE INHIBITOR functions in resistance to necrotrophic fungi and insect herbivory. Plant J. 68, 480-494.

Lee, D.-S., Lee, K.-H., Jung, S., Jo, E.-J., Han, K.-H. and Bae, H.-J. (2012) Synergistic effects of $2 \mathrm{~A}$-mediated polyproteins on the production of lignocellulose degradation enzymes in tobacco plants. J. Exp. Bot. 63 , 4797-4810.

Ma, C.L. and Mitra, A. (2002) Expressing multiple genes in a single open reading frame with the $2 \mathrm{~A}$ region of foot-and-mouth disease virus as a linker. Mol. Breeding 9, 191-199.

Marino-Buslje, C., Venhudová, G., Molina, M.A., Oliva, B., Jorba, X., Canals, F., Avilés, F.X. and Querol, E. (2000) Contribution of C-tail residues of potato carboxypeptidase inhibitor to the binding to carboxypeptidase A. Eur. J. Biochem. 267, 1502-1509.

Miranda, R., Zamudio, F.Z. and Bravo, A. (2001) Processing of Cry1Ab delta-endotoxin from Bacillus thuringiensis by Manduca sexta and Spodoptera frugiperda midgut proteases: role in protoxin activation and toxin inactivation. Insect Biochem. Mol. Biol. 31, 1155-1163.

Moreno, A.B., Peñas, G., Rufat, M., Bravo, J.M., Estopà, M., Messeguer, J. and San Segundo, B. (2005) Pathogen-induced production of the antifungal AFP protein from Aspergillus giganteus confers resistance to the blast fungus Magnaporthe grisea in transgenic rice. Mol. Plant-Microbe Interact. 18, 960-972.

Outchkourov, N.S., de Kogel, W.J., Wiegers, G.L., Abrahamson, M. and Jongsma, M.A. (2004) Engineered multidomain cysteine protease inhibitors yield resistance against western flower thrips (Franklinielia occidentalis) in greenhouse trials. Plant Biotechnol. J. 2, 449-458.

Park, S., Kang, K., Kim, Y.S. and Back, K. (2009) Endosperm-specific expression of tyramine $\mathrm{N}$-hydroxycinnamoyltransferase and tyrosine decarboxylase from a single self-processing polypeptide produces high levels of tyramine derivatives in rice seeds. Biotechnol. Lett. 31, 911-915.

Quilis, J., Meynard, D., Vila, L., Avilés, F.X., Guiderdoni, E. and San Segundo, B. (2007) A potato carboxypeptidase inhibitor gene provides pathogen resistance in transgenic rice. Plant Biotechnol. J. 5, 537-553.

Ralley, L., Enfissi, E.M.A., Misawa, N., Schuch, W., Bramley, P.M. and Fraser, P.D. (2004) Metabolic engineering of ketocarotenoid formation in higher plants. Plant J. 39, 477-486.

Ryan, C.A. (1990) Protease Inhibitors in Plants: Genes for Improving Defenses Against Insects and Pathogens. Annu. Rev. Phytopathol. 28, 425-449.

Sanahuja, G., Banakar, R., Twyman, R.M., Capell, T. and Christou, P. (2011) Bacillus thuringiensis: a century of research, development and commercial applications. Plant Biotechnol. J. 9, 283-300.

Schluter, U., Benchabane, M., Munger, A., Kiggundu, A., Vorster, J., Goulet, M.C., Cloutier, C. and Michaud, D. (2010) Recombinant protease inhibitors for herbivore pest control: a multitrophic perspective. J. Exp. Bot. 61, 4169-4183.

Senthilkumar, R., Cheng, C.P. and Yeh, K.W. (2010) Genetically pyramiding protease-inhibitor genes for dual broad-spectrum resistance against insect and phytopathogens in transgenic tobacco. Plant Biotechnol. J. 8, 65-75.

Srinivasan, A., Giri, A.P., Harsulkar, A.M., Gatehouse, J.A. and Gupta, V.S. (2005) A Kunitz trypsin inhibitor from chickpea (Cicer arietinum L.) that exerts anti-metabolic effect on podborer (Helicoverpa armigera) larvae. Plant Mol. Biol. 57, 359-374. 
Stevens, J., Dunse, K., Fox, J., Evans, S. and Anderson, M. (2012) Biotechnological Approaches for the Control of Insects Pests in Crop Plants. In: Pesticides - Advances in Chemical and Botanical Pesticides (Soundararajan, R.P., ed.) pp. 269-308. Rijeka, Croatia: InTech.

Talbot, N.J. (2003) On the trail of a cereal killer: exploring the biology of Magnaporthe grisea. Annu. Rev. Microbiol. 57, 177-202.

Tamayo, M.C., Rufat, M., Bravo, J.M. and San Segundo, B. (2000) Accumulation of a maize proteinase inhibitor in response to wounding and insect feeding, and characterization of its activity toward digestive proteinases of Spodoptera littoralis larvae. Planta 211, 62-71.

Vila, L., Quilis, J., Meynard, D., Breitler, J.C., Marfà, V., Murillo, I., Vassal, J.M., Messeguer, J., Guiderdoni, E. and San Segundo, B. (2005) Expression of the maize proteinase inhibitor $(\mathrm{mpl})$ gene in rice plants enhances resistance against the striped stem borer (Chilo suppressalis): effects on larval growth and insect gut proteinases. Plant Biotechnol. J. 3, 187-202.

Villanueva, J., Canals, F., Prat, S., Ludevid, D., Querol, E. and Avilés, F.X. (1998) Characterization of the wound-induced metallocarboxypeptidase inhibitor from potato - cDNA sequence, induction of gene expression, subcellular immunolocalization and potential roles of the C-terminal propeptide. FEBS Lett. 440, 175-182

Zavala, J.A., Patankar, A.G., Gase, K. and Baldwin, I.T. (2004) Constitutive and inducible trypsin proteinase inhibitor production incurs large fitness costs in Nicotiana attenuata. Proc. Natl Acad. Sci. USA 101, 1607-1612.

\section{Supporting information}

Additional Supporting information may be found in the online version of this article:

Figure S1 Southern blot analysis of the mpi-pci transgenic lines (T0 generation).

Figure S2 Growth of Chilo suppressalis larvae fed on transgenic rice plants (T2 homozygous generation) expressing a mpi-pci fusion gene under the control of the mpi promoter.

Figure S3 Immunodetection of the MPI protein in rice plants expressing the mpi-2A-pci gene under the control of the mpi promoter

Table S1 Oligonucleotide primer sequences used in this study.

Methods S1 Construction of plant expression vectors, rice transformation, wounding of plant leaves, immunoblot analysis, insect bioassays and blast resistance assays. 